\title{
Searching for gravity assisted trajectories to accessible near-Earth asteroids
}

\author{
Ştefan Berinde \\ Babes-Bolyai University, Cluj-Napoca, Romania \\ email: sberinde@math.ubbcluj.ro
}

\begin{abstract}
This paper explores the advantages of using gravity assisted trajectories to perform flyby and rendezvous missions to accessible near-Earth asteroids (NEAs), in terms of the total velocity budget required for the mission. Combining the Opik's formalism of close encounters with the Monte Carlo sampling technique and Lambert trajectories, we give a general picture of the accessibility regions for NEAs in phase space of orbital elements, without considering the phasing requirements between bodies.
\end{abstract}

Keywords. Gravity assisted trajectories, flyby missions, near-Earth asteroids

\section{Introduction}

Given the increased interest for in-situ exploration of near-Earth asteroids (NEAs) in recent years, several papers explored already the possibility of direct transfer orbits for flyby and rendezvous missions (Perozzi, Rossi \& Valsecchi 2001, Christou 2003). As we will highlight in this paper, the accessibility region in phase space of orbital elements is rather limited for such missions due to the high velocity budget involved.

Without considering the phasing requirements (proper alignment of the bodies on their orbits), which is just a matter of timing, this paper gives a general overview on the advantages of using gravity assisted trajectories to NEAs. The key parameters used to describe the accessibility regions are the nodal distances, eccentricity and inclination of the asteroid's orbit. Figure 1 shows the distribution of discovered NEAs in terms of these parameters. A strong observational bias near the Earth's orbit is evident.
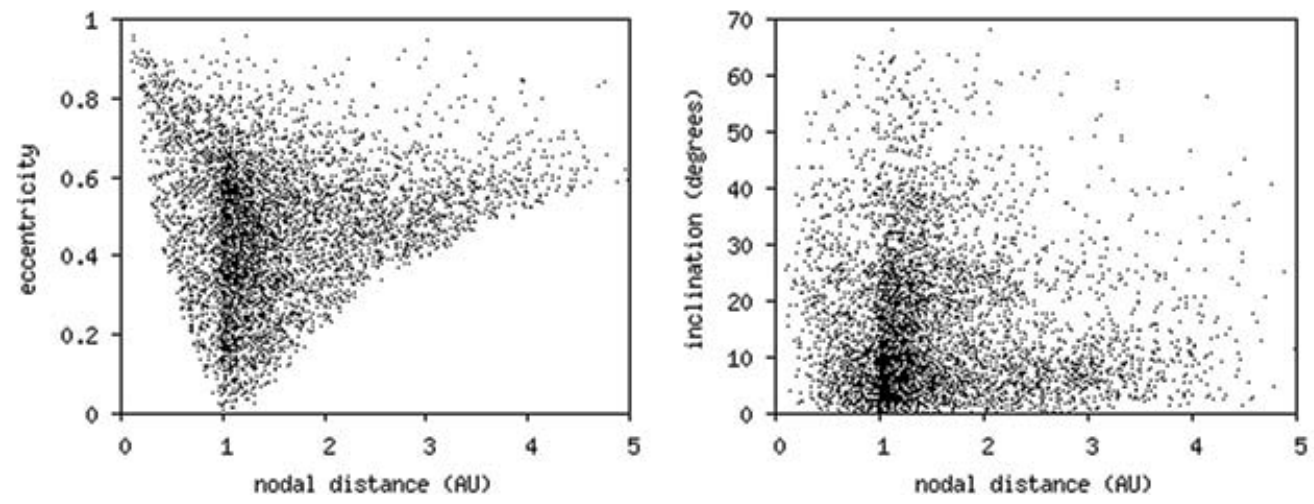

Figure 1. Distribution of discovered NEAs in nodal distance-eccentricity plane (left panel) and in nodal distance-inclination plane (right panel). Both nodal distances are considered for each asteroid. 


\section{Preliminaries}

Throughout this paper we adopt the following measuring units for distance and time, such that the radius of the Earth's orbit (supposed to be circular) is 1 and its heliocentric velocity is also 1 . It means that the heliocentric gravitational parameter $\mu_{\odot}=1$.

We consider the initial state of an interplanetary spacecraft as being its parking LEO orbit (at about $200 \mathrm{~km}$ ). From here, we will try to evaluate the velocity budget $d v$ required to place the spacecraft in a flyby or rendezvous orbit with an asteroid. Let $d v_{0}$ be the excess velocity applied in LEO orbit. The unperturbed geocentric relative velocity $u_{0}$ (that is, velocity at infinity) is given by

$$
u_{0}=\sqrt{\left(v_{l e o}+d v_{0}\right)^{2}-v_{\text {par }}^{2}},
$$

where $v_{l e o}=0.26$ is the circular velocity in LEO orbit and $v_{p a r}=0.37$ is the corresponding parabolic velocity. For escaping the Earth, we must have $d v_{0}>0.11$. We also note that $u_{0}>d v_{0}$ only for $d v_{0}>0.13$. For practical reasons we can suppose that $d v_{0}<0.2$, since the total $d v$ budget for NEAs missions is rather limited $(6 \mathrm{~km} / \mathrm{s}$ for the NEAR mission; see Farquhar et al. 1995).

Also in this section we will reproduce the formulas for the components of the heliocentric velocity vector of a body in an elliptic orbit, at one of its nodes. This will prove useful later when computing the excess velocity $d v_{\text {rend }}$ required to perform a rendezvous mission at the nodes. Let be a rectangular reference frame oriented such that the $y$-axis points along the radial distance of the body towards the Sun, the $z$-axis is perpendicular on the ecliptic plane towards north pole and the $x$-axis completes the right-handed frame. Because we are at the nodes the $x$-axis is located on the ecliptic plane and we must have $v_{x}=\sqrt{v_{x}^{2}+v_{z}^{2}} \cos i$ and $v_{z}= \pm \sqrt{v_{x}^{2}+v_{z}^{2}} \sin i$. Writing the component $v_{y}$ (the radial velocity) in terms of the keplerian orbital elements $a, e, i$, we get the final expressions

$$
\left\{\begin{array}{l}
v_{x}=\frac{\sqrt{a}}{d} \sqrt{1-e^{2}} \cos i \\
v_{y}= \pm \frac{\sqrt{a}}{d} \sqrt{e^{2}-\left(1-\frac{d}{a}\right)^{2}} \\
v_{z}= \pm \frac{\sqrt{a}}{d} \sqrt{1-e^{2}} \sin i
\end{array}\right.
$$

where $d$ is the nodal distance. The signs of the right terms depend on the type of the node, if it is ascending or descending (for the component $v_{z}$ ) and if it is placed before or after the perihelion (for the component $v_{y}$ ).

\section{Direct orbits}

Following the basic optimal guidelines about orbital transfer in space (Marinescu 1982), a rendezvous mission with an asteroid includes, in general, two steps: a Hohmann transfer orbit from Earth to the farthest node of the asteroid's orbit, followed by a velocity impulse in order to match its orbital velocity.

A nodal distance $d$ can be reached at aphelion on an orbit with the initial geocentric relative velocity $u_{0}$, along the Earth's orbit, given by

$$
u_{0}=\sqrt{\frac{2 d}{(1+d)}}-1 .
$$


The velocity at aphelion will be

$$
v_{a p h}=\sqrt{\frac{2}{d(1+d)}} .
$$

In the previously introduced reference frame, this velocity is oriented along the $x$-axis. Using now the set of formulas (2.2), we obtain the required rendezvous velocity impulse at the node

$$
d v_{\text {rend }}^{2}=\frac{2(2+d)}{d(1+d)}-\frac{1}{a}-\frac{2}{d} \sqrt{\frac{2 a\left(1-e^{2}\right)}{d(1+d)}} \cos i,
$$

where $a, e, i, \omega$ are the well known orbital elements of the asteroid. Note that we must have

$$
d=\frac{a\left(1-e^{2}\right)}{1 \pm e \cos \omega}
$$

where the sign depends on the node's type.

From (2.1) and (3.1) we can get $d v_{0}$ in terms of $d$. Then $d v=d v_{0}+d v_{\text {rend }}$ is the total velocity budget to accomplish the mission. Based on this approach, figure 2 gives the accessibility regions for such missions in the $d$-e plane. The required rendezvous velocity is minimal for $\omega=0$ and $\omega=\pi$ and maximal for $\omega=\pi / 2$ and $\omega=3 \pi / 2$. It depends also strongly on the orbital inclination $i$, such that only orbits very close to the ecliptic are matched within the limit $d v<0.2$.

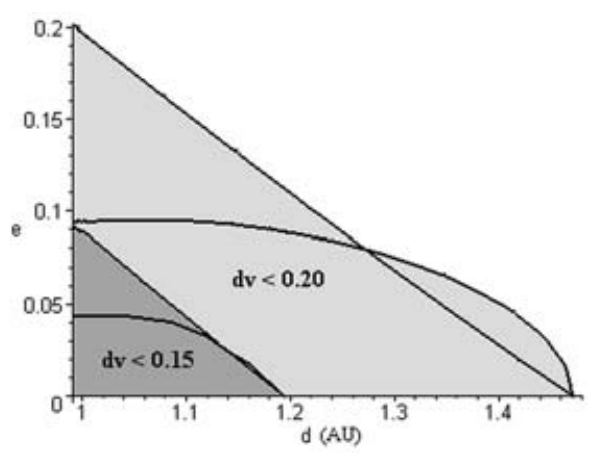

Figure 2. Accessibility regions in $d$-e plane for a rendezvous mission with an asteroid (see text for details). Four regions are depicted, depending on the total $d v$ budget and on the perihelion argument of the orbit ( $\omega=0$ for straight borders and $\omega=\pi / 2$ for curved borders). We set here $i=0$.

As a matter of fact, if the asteroid's orbit intersect the Earth's one $\left(d=1, u_{0}=0\right)$, we get the well known formula (Carusi et al. 1990)

$$
d v_{\text {rend }}^{2}=3-\frac{1}{a}-2 \sqrt{a\left(1-e^{2}\right)} \cos i=3-T,
$$

where $d v_{\text {rend }}$ has now the meaning of the unperturbed geocentric relative velocity and $T$ is the Tisserand parameter.

It follows that the accessibility region of NEAs for rendezvous missions on direct orbits is very limited. If we consider only the flyby missions opportunities at the nodes, all NEAs are reachable since they have (by definition) at least one of their nodal distances between 1.0 and $1.3 \mathrm{AU}$. 


\section{VEGA and VEEGA gravity assisted orbits}

In this section we want to explore the advantages of the gravity assisted orbits to the NEAs. It is practically impossible to give a general picture about the entire spectrum of gravity assisted orbits in the inner solar system. But, if we neglect the possibility of intermediary deep-space maneuvers involving significant velocity impulses (which was not the case for the NEAR mission; Farquhar et al. 1995), the only ways to increase orbital energy in a reasonable amount of time are VEGA (Venus-Earth Gravity Assist) and VEEGA (Venus-Earth-Earth Gravity Assist) transfer orbits (Longuski \& Williams 1991).

Next we will work in the frame of Opik's geometric formalism (Carusi et al. 1990). This gives us the opportunity to develop simple algebraic formulas for various orbital quantities from the beginning of the mission (LEO orbit) till the end (asteroid flyby or rendezvous). We recall some of the principles of this approximation: planetary orbits are considered circular and coplanar, the spacecraft has a planetocentric hyperbolic orbit near a planet and a keplerian heliocentric orbit in interplanetary space and a planetary encounter is considered an instantaneous event when compared with the interplanetary travel time.

To perform an interplanetary journey Earth-Venus-Earth, the alignment of these two planets and the initial conditions are very sensitive to each other. Since we do not take into consideration here the phasing requirements, we focus on the initial conditions for VEGA orbital transfers.

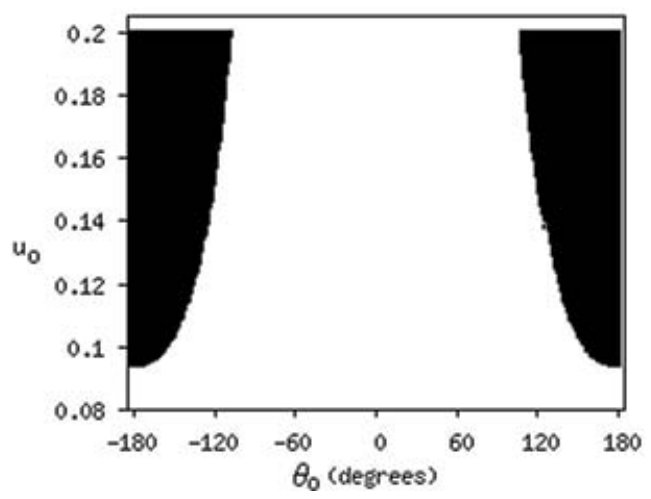

Figure 3. All possible starting conditions from Earth $\left(\theta_{0}, u_{0}\right)$ for VEGA transfer orbits.

Let $\theta_{0}$ denote the angle between the Earth's velocity vector and the initial geocentric relative velocity vector of the spacecraft (with magnitude $u_{0}$ ). We consider this angle to be negative if the relative velocity vector points inside the Earth's orbit. The heliocentric starting conditions for a coplanar transfer are fully described by the pair $\left(\theta_{0}, u_{0}\right)$. Figure 3 shows all the pairs for which a VEGA orbit exists. These solutions were computed by Monte Carlo sampling technique, solving the Lambert problem twice and patching the conic sections at Venus encounter Battin (1987). This encounter has the effect of deflecting the spacecraft relative velocity vector $u$ by the amount

$$
\sin \frac{\gamma}{2}=\left(1+\frac{\rho}{m_{p}} u^{2}\right)^{-1},
$$

where $\rho$ is the minimum encounter distance and $m_{p}$ the planetary mass. The angular 
deflection $\gamma$ must not exceed $\gamma_{\max }$, obtained from (4.1) when $\rho$ equals the Venus planetary radius.

If we are interested in maximizing the relative velocity of the spacecraft $u$ when it encounters the Earth again, for each value of $u_{0}$ we can find the right value of $\theta_{0}$ for which the maximum occurs. Figure 4 presents the relation between $u$ and $u_{0}$. An empirical linear dependence among these parameters is also determined. In these conditions, the spacecraft will encounter Earth at an angle $\theta \in\left(94^{\circ}, 99^{\circ}\right)$, defined analogously to $\theta_{0}$.

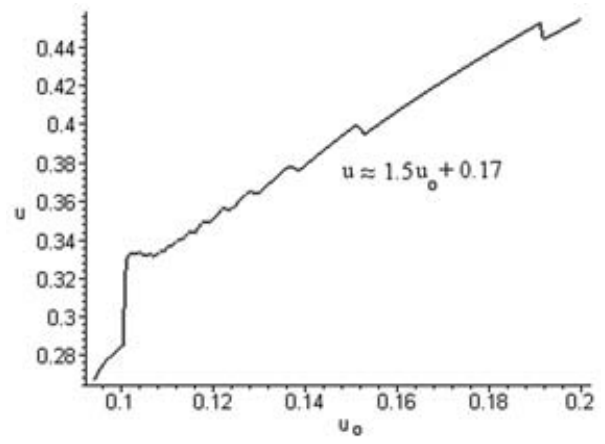

Figure 4. Maximum relative velocity $u$ at Earth encounter for VEGA transfer orbits. An empirical linear dependence can be inferred between the initial and final relative velocities: $u \approx 1.5 u_{0}+0.17$, valid for $u_{0}>0.1$.

If we define $\gamma_{\max }$ as the maximum angular deflection at Earth encounter (when the geocentric minimum distance $\rho$ equals the radius of the LEO orbits), we get an approximate expression for it, $\sin \left(\gamma_{\max } / 2\right) \approx\left(1+14.5 u^{2}\right)^{-1}$. This value imposes a threshold limit for the final orbital energy acquired in VEGA transfer orbits. But, since the relative velocity $u$ and angle $\theta$ are rather large after this encounter, an additional Earth encounter allows a further variation of the semimajor axis of the spacecraft's orbit. In other words, the spacecraft must enter in a mean motion resonance with the Earth, on a VEEGA transfer orbit. If we set up a resonance ratio $p / q$ and a corresponding semimajor axis of the post-encounter orbit $a=(q / p)^{2 / 3}$, there is a precise deflection angle $\gamma_{r e s}$ for which the resonance is matched. Its value is given by $\gamma_{r e s}=\theta-\theta^{\prime}$, where $\theta^{\prime}$ is computed from

$$
a=\frac{1}{1-2 u \cos \theta^{\prime}-u^{2}},
$$

a well known expression for the semimajor axis in the Opik's formalism. We found that the optimum low-order mean motion resonances are $1 / 2$ and $2 / 5$. The corresponding deflection angles $\gamma_{1 / 2}$ and $\gamma_{2 / 5}$ are computed in figure 5 , for each value of the initial relative velocity $u_{0}$.

Besides the post-encounter semimajor axis $a$ of the spacecraft's orbit, its eccentricity $e$ can be also computed from

$$
e=\sqrt{1-\frac{1}{4 a}\left(3-\frac{1}{a}-u^{2}\right)^{2}},
$$

and so we can get the aphelion distance $d$ for the final VEGA and VEEGA transfer orbits. Figure 6 gives these values computed with the condition $\theta^{\prime}=\theta-\gamma_{\max }$ (for VEGA orbits) and with the condition $\theta^{\prime}=\theta-\gamma_{1 / 2}-\gamma_{\max } / 2$ for VEEGA orbits. For the last type of orbits, the second angular deflection was limited at $\gamma_{\max } / 2$, since a higher value will send the spacecraft beyond Jupiter. 


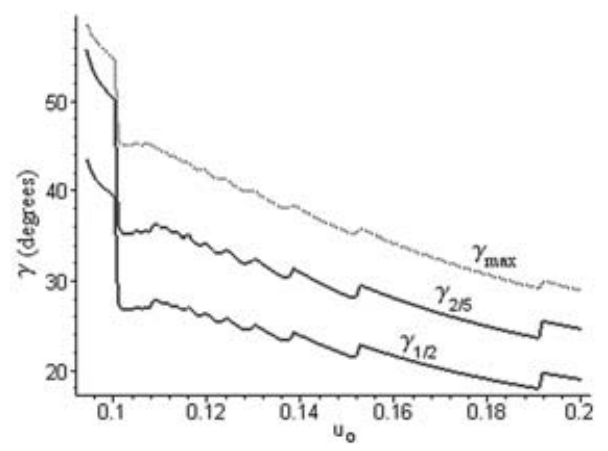

Figure 5. Angular deflection of the velocity vector at Earth encounter for a given initial relative velocity $u_{0} \cdot \gamma_{\max }$ - maximum deflection, $\gamma_{2 / 5}$ - required deflection to set up an orbit in $2 / 5$ mean motion resonance with the Earth, $\gamma_{1 / 2}$ - required deflection to set up an orbit in $1 / 2$ mean motion resonance with the Earth.

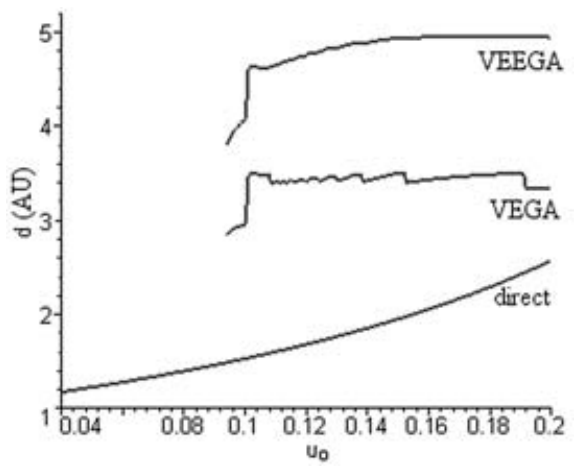

Figure 6. Maximum aphelion distance $d$, function of the initial relative velocity $u_{0}$, for different transfer orbits: direct orbits, VEGA orbits (computed for $\gamma=\gamma_{\max }$ ) and VEEGA orbits (computed for $\left.\gamma=\gamma_{1 / 2}+\gamma_{\max } / 2\right)$.

As we can see from Figures 1 and 6 , with a low initial velocity $u_{0}$ a spacecraft can reach both orbital nodes of the majority of the NEAs. The existence of such an orbit depends only on the phasing requirements. This permits a flyby at the farthest orbital node of an asteroid's orbit, where a rendezvous maneuver requires less additional velocity.

\section{References}

Battin, R.H. 1987, An introduction to the mathematics and methods of astrodynamics, AIAA Education Series, New-York

Carusi, A., Valsecchi, G.B. \& Greenberg R. 1990, Cel. Mech. Dyn. Astron. 49, 111

Christou, A. 2003, Planet. Space Sci. 51, 221

Farquhar, R.W., Dunham, D.W. \& McAdams J.V. 1995, J. Astronautical Sci. 43, 353

Longuski, J.M. \& Williams S.N. 1991, Cel. Mech. Dyn. Astron. 52, 207

Marinescu, A. 1982, Optimal problems in the dynamics of space flight (in romanian), Editura Academiei, Bucuresti

Perozzi, E., Rossi, A. \& Valsecchi G.B. 2001, Planet. Space Sci. 49, 3 